Pacific Journal of Mathematics

ON INDECOMPOSABLE MODULES OVER RINGS WITH 


\title{
ON INDECOMPOSABLE MODULES OVER RINGS WITH MINIMUM CONDITION
}

\author{
R. R. Colby
}

Let $A$ be an associative ring with left minimum condition and identity. Let $g(d)$ denote the number of nonisomorphic indecomposable $A$-modules which have composition length $d, d$ a nonnegative integer. If, for each integer $n$, there exists an integer $d>n$, such that $g(d)=\infty, A$ is said to be of strongly unbounded module type.

Assume that the center of the endomorphism ring of each simple (left) $A$-module is infinite. The following results concerning the structure of rings of strongly unbounded type are obtained.

I. If the ideal lattice of $A$ is infinite, then $A$ is of strongly unbounded module type.

II. If $A$ is commutative, then $A$ has only a finite number of (nonisomorphic) finitely generated indecomposable modules if and only if the ideal lattice of $A$ is distributive. Otherwise, $A$ is of strongly unbounded module type.

III. If the ideal lattice of $A$ contains a vertex $V$ of order greater than three such that, for some primitive idempotent $e \in A$, the image $V e$ of $V$ is a vertex of order greater than three in the submodule lattice of $A e$, then $A$ is of strongly unbounded module type.

These results are generalizations of earlier ones obtained by J. P. Jans for finite dimensional algebras over algebraically closed fields.

Let $A$ be an associative ring with left minimum condition and identity. The length, $c(M)$, of a (left) $A$-module $M$ with composition series is the number of composition factors of $M$. Let $g(d)$ denote the number of nonisomorphic indecomposable $A$-modules which have length $d, d$ a nonnegative integer. If $\sum_{d} g(d)<\infty, A$ is said to be of finite module type. If there exists an integer $n$ such that $g(d)=0$ for all $d>n, A$ is of bounded module type. If not of bounded module type, $A$ is of unbounded module type. If for each integer $n$, there exists $d>n$ such that $g(d)=\infty, A$ is of strongly unbounded module type. R. Brauer, J. P. Jans, and R. M. Thrall have conjectured that infinite algebras of unbounded type are of strongly unbounded type, and that algebras of bounded type are of finite type [4]. A discussion of the state of these conjectures may be found in [2] and [4].

J. P. Jans has given sufficient conditions that a finite dimensional algebra over an algebraically closed field be of strongly unbounded type [4]. Through extension and modification of the techniques used 
by Jans and by H. Tachikawa [6], some of these results can be obtained for arbitrary rings with minimum condition, provided that the endomorphism rings of the simple $A$-modules have infinite centers.

2. Rings with infinite ideal lattices. Let $A$ be a ring with left minimum condition with the property that the lattice of ideals of $A$ is infinite. H. Tachikawa showed that $A$ is of unbounded type [6]. If $A$ is also a finite dimensional algebra over an algebraically closed field, $A$ is of strongly unbounded type [4]. The following theorem generalizes these results.

THEOREM. If the center of the endomorphism ring of each simple (irreducible) A-module is infinite and if the ideal lattice of $A$ is infinite, then $A$ is of strongly unbounded module type.

Proof. Since the ideal lattice of $A$ is infinite, the lattice contains a projective root [1].

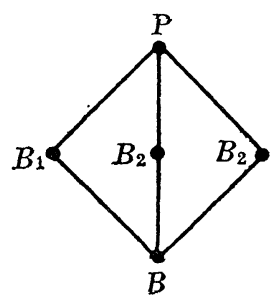

Since $A / B$-modules are $A$-modules, we can assume that $B=0$. Also, there exists an $A-A$ isomorphism $\psi: B_{1} \cong B_{2}$. Let $N$ denote the radical of $A$ and define $M=l(N) \cap r(N)$. Since $B_{1}$ and $B_{2}$ are simple ideals we have $B_{1}+B_{2}=B_{1} \oplus B_{2} \subseteq M$. There exist primitive idempotents $e, f \in A$ such that $f M e \supseteqq f B_{1} e \oplus f B_{2} e \supset(0)$. Choose $u=$ $f u e \neq 0$ in $f B_{1} e$ and let $v=\psi(u)$. Let $\Lambda \subset f A f$ be a set of representatives for the nonzero distinct cosets of the center of $f A f / f N f$. Evidently, $\Lambda$ is infinite. For $\lambda \in \Lambda$, define $s(\lambda)=\lambda v-u$. Since $f A u$, $f A v, f A s(\lambda)$, are all nonzero and $u, v, s(\lambda) \in M$, we have $A f / N f \cong$ $A u \cong A v \cong A s(\lambda)$.

LeMma 1. If $\lambda \neq \mu \in \Lambda, a, b \in A$, and $s(\lambda) a=b s(\mu)$, then $s(\lambda) a=$ $b s(\mu)=0$.

Proof. We may assume that $a \in e A e, b \in f A f$. Since $B_{1} \cap B_{2}=0$, we have $\lambda v a=b \mu v$ and $u a=b u$. Since $v=\psi(u), v a=b v$ so that $\lambda b v=$ $b \mu v$. Thus, since $f A f / f N f$ is a division ring, $\lambda b=b \mu(\bmod f N f)=$ $\mu b(\bmod f N f)$. Since $\lambda \neq \mu(\bmod f N f), b=0(\bmod f N f)$. Since $v \in M$, the lemma follows. 
Lemma 2. If $a, b, c, d \in A$ and $s(\lambda) a+v b=c s(\lambda)+d v$, then $v a=$ $c v, u a=c u$, and $v b=d v$.

Proof. Since $B_{1} \cap B_{2}=0$ and $v=\psi(u)$, we have $c u=u a, c v=$ $v a$, and $\lambda v a+v b-c \lambda v-d v=0$. Hence, since $\lambda c=c \lambda(\bmod f N f)$, $v b=d v$.

For each positive integer $n$, let $X^{n}$ be the direct sum of $n$ copies of $A e$,

$$
X^{n}=\bigoplus \sum_{i=1}^{n} \varepsilon_{i}(A e),
$$

and let $Y^{n}$ denote the socle of $X^{n}$. For $\lambda \in \Lambda$, define

$$
T_{\lambda}^{n}=\left\{\sum_{i=1}^{n} \varepsilon_{i}\left(a_{i-1} v+a_{i} s(\lambda)\right): a_{0}=0, a_{i} \in A\right\} .
$$

Let $H_{\lambda}^{n}=X^{n} / T_{\lambda}^{n}$ and $S_{\lambda}^{n}=Y^{n} / T_{\lambda}^{n}$. Since the length of $T_{\lambda}^{n}$ is $n$, the length of $S_{\lambda}^{n} \geqq 2 n-n=n$.

We proceed to show that $H_{\lambda}^{n}$ and $H_{\mu}^{n}$ are not isomorphic, provided $\lambda \neq \mu \in \Lambda$. Suppose $\theta: H_{\lambda}^{n} \cong H_{\mu .}^{n}$. Since $X^{n}$ is projective [3], there exists $\bar{\theta}: X^{n} \rightarrow X^{n}$ such that $\theta \pi_{\lambda}=\pi_{\mu} \bar{\theta}$, where $\pi_{\lambda}, \pi_{\mu}$ are the natural projections of $X^{n}$ onto $H_{\lambda}^{n}, H_{\mu}^{n}$, respectively. There exist $x_{1}, \cdots, x_{n} \in e A e$, such that

$$
\bar{\theta} \varepsilon_{n}(e)=\sum_{i=1}^{n} \varepsilon_{i}\left(x_{i}\right)
$$

Since $\pi_{\lambda} \varepsilon_{n} s(\lambda)=0$, and $\theta \pi_{\lambda}=\pi_{\mu} \bar{\theta}$, we have $\pi_{\mu} \bar{\theta} \varepsilon_{n} s(\lambda)=0$ and hence $\bar{\theta} \varepsilon_{n} s(\lambda) \in T_{\mu}^{n}$. Thus,

$$
\sum_{i=1}^{n} \varepsilon_{i}\left(s(\lambda) x_{i}\right)=\bar{\theta} \varepsilon_{n} s(\lambda) \in T_{\mu}^{n} .
$$

According to the definition of $T_{\mu}^{n}$, there exist $a_{0}=0, a_{1}, \cdots, a_{n} \in A$, such that

$$
s(\lambda) x_{i}=a_{i-1} v+a_{i} s(\mu), \quad i=1, \cdots, n .
$$

Using an induction and Lemma 1 , we conclude that $x_{1}, \cdots, x_{n} \in e N e$, and hence

$$
\theta \pi_{\lambda} \varepsilon_{n}(v)=\pi_{\mu} \sum_{i=1}^{n} \varepsilon_{i}\left(v x_{i}\right)=0
$$

This contradicts the assumption that $\theta$ is an isomorphism.

Next, suppose that $H_{\lambda}^{n}$ decomposes. Let $\eta$ be the idempotent endomorphism of $H_{\lambda}^{n}$ associated with an indecomposable direct summand of $H_{\lambda}^{n}$ such that $\eta \pi_{\lambda} \varepsilon_{n}(v) \neq 0$. 
LeMma 3. The restriction of $\eta$ to $S_{\lambda}^{n}$ is a monomorphism.

Proof. Since $X^{n}$ is projective, $\eta$ may be lifted to an endomorphism $\bar{\eta}$ of $X^{n}$. There exist $y_{i j} \in e A e$ such that

$$
\bar{\eta} \varepsilon_{j}(e)=\sum_{i=1}^{n} \varepsilon_{i}\left(y_{i j}\right), \quad j=1, \cdots, n .
$$

From the definition of $T_{\lambda}^{n}$, we have that

$$
\bar{\eta}\left(\varepsilon_{j-1}(s(\lambda))+\varepsilon_{j}(v)\right) \in T_{\lambda}^{n}, \quad j=2, \cdots, n .
$$

and $\bar{\eta} \varepsilon_{n}(s(\lambda)) \in T_{\lambda}^{n}$. Thus,

$$
\bar{\eta}\left(\varepsilon_{j-1}(s(\lambda))+\varepsilon_{j}(v)\right)=\sum_{i=1}^{n} \varepsilon_{i}\left(s(\lambda) y_{i, j-1}+v y_{i j}\right) \in T_{\lambda}^{n},
$$

for $j=2, \cdots, n$, and

$$
\bar{\eta} \varepsilon_{n} s(\lambda)=\sum_{i=1}^{n} \varepsilon_{i}\left(s(\lambda) y_{i n}\right) \in T_{\lambda}^{n} .
$$

Hence, there exist $a_{i j} \in f A f$ such that

$$
\begin{aligned}
s_{\lambda} y_{1, j-1}+v y_{1 j} & =a_{1, j-1} s_{\lambda}, \\
s_{\lambda} y_{i, j-1}+v y_{i j} & =a_{i, j-1} s_{\lambda}+a_{i-1, j-1} v, \\
s_{\lambda} y_{1 n} & =a_{1 n} s_{\lambda},
\end{aligned}
$$

and

$$
\begin{aligned}
& s_{\lambda} y_{i n}=a_{i n} s_{\lambda}+a_{i-1, n} v, \\
& \text { for } i, j=2,3, \cdots, n \text {. }
\end{aligned}
$$

Since $f s_{\lambda} e=s_{\lambda}$ and $f v e=v$, we may assume that $a_{i j} \in f A f, i, j=$ $1,2, \cdots, n$. Applying Lemma 2, we obtain,

$$
u y_{i j}=a_{i j} u,
$$

and

$$
\begin{array}{ll}
v y_{i j}=a_{i j} v, & i, j=1,2, \cdots, n ; \\
v y_{i j}=a_{i-1, j-1} v, & i, j=2,3, \cdots, n ;
\end{array}
$$

and

$$
y_{i-1, n}=0(\bmod e N e), \quad i=2,3, \cdots, n .
$$

Suppose $i<j$. Then we have

$$
v y_{i j}=a_{i j} v=v y_{i+1, j+1}=\cdots=v y_{i+n-j, n}=0 .
$$

Therefore, $y_{i j}=0(\bmod e N e)$. Suppose $i>j$. Then 


$$
v y_{i j}=a_{i-1, j-1} v=v y_{i-1, j-1}=\cdots=v y_{i-j+1,1} .
$$

Also,

$$
v y_{k k}=v y_{n n}, \quad k=1,2, \cdots, n .
$$

Since $\eta \pi_{\lambda}\left(\varepsilon_{n}(v)\right)=\pi_{\lambda} \varepsilon_{n}\left(v y_{n n}\right) \neq 0$, we have $y_{n n} \neq 0(\bmod e N e)$. From these equations and the idempotence of $\eta$ it follows that

$$
y_{i j}= \begin{cases}e(\bmod e N e), & \text { if } i=j \\ 0(\bmod e N e), & \text { if } i<j \\ y_{i-j+1,1}(\bmod e N e), & \text { if } i>j\end{cases}
$$

Next assume that $x \in Y^{n}$ and $\eta \pi_{\lambda}(x)=0$. Then $\bar{\eta}(x) \in T_{\lambda_{0}}^{n}$. There exist elements $r_{j}$ of the socle of $A e$ such that $x=\sum_{j=1}^{n} \varepsilon_{j} r_{j}$, from which the equation

$$
\bar{\eta}(x)=\sum_{i=1}^{n} \varepsilon_{i}\left(r_{i}+\sum_{j=1}^{i-1} r_{j} y_{i-j+1,1}\right)
$$

follows. Since $\bar{\eta}(x) \in T_{\lambda}^{n}$, there exist $b_{0}=0, b_{1}, \cdots, b_{n} \in A e$ such that

$$
\sum_{j=1}^{i-1} r_{j} y_{i-j+1,1}+r_{i}=b_{i} s(\lambda)+b_{i-1} v, \quad i=2, \cdots, n .
$$

Defining

$$
\begin{aligned}
& \alpha_{0}=0, \\
& \alpha_{1}=b_{1}, \\
& \alpha_{k}=b_{k}-\sum_{j=1}^{k-1} \alpha_{j} \alpha_{k-j+1,1}, \quad k=2, \cdots, n .
\end{aligned}
$$

it follows that

$$
r_{k}=\alpha_{k} s(\lambda)+\alpha_{k-1} v, \quad k=1, \cdots, n .
$$

Thus, $x \in T_{\lambda}^{n}$ and $\pi_{\lambda} x=0$. This proves Lemma 3 .

From Lemma 3, we conclude that $S_{\lambda}^{n}$ is contained in an idecomposable direct summand $V_{\lambda}$ of $H_{\lambda}^{n}$. Calculation of $H_{\lambda}^{n} / S_{\lambda}^{n} \cong X^{n} / Y^{n}$ shows that every direct summand of $H_{\lambda}^{n}$ not equal to $V_{\lambda}$ is isomorphic to $A e / S(A e), S(A e)$ the socle of $A e$. Thus, $V_{\lambda} \cong V_{\mu}$ if and only if $H_{\lambda}^{n} \cong H_{\mu}^{n}$ and hence $V_{\lambda} \not V_{\mu}$ if $\lambda \neq \mu \in \Lambda$. This completes the proof of the theorem.

\section{Commutative rings.}

THeORem. If $A$ is commutative, then $A$ is of finite type if and only if the ideal lattice of $A$ is distributive. Otherwise, $A$ is of 
unbounded type, strongly so if the endomorphism ring of each simple A-module is infinite.

Proof. It is sufficient to show that, if the ideal lattice of $A$ is distributive, $A$ is generalized uni-serial (see [5]). Let $e$ be a primitive idempotent in $A$ and consider the lattice of submodules of $A e$. Since $A$ is commutative, these submodules are ideals in $A$. Suppose the lattice contains a vertex

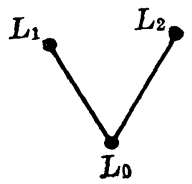

where we assume, without loss of generality, that the lattice from (0) to $L_{0}$ is a chain. Then $L_{0}=N^{k+1} e$ for some $k$, and $L_{1}+L_{2} \subseteq N^{k} e$. Choose $\alpha_{1} \in L_{i}-L_{0}, i=1,2$, and define

$$
L_{3}=A e\left(\alpha_{1}+\alpha_{2}\right)+L_{0} \text {. }
$$

The mapping $a e \rightarrow a e\left(\alpha_{1}+\alpha_{2}\right)+L_{0}$ induces an isomorphism $L_{3} / L_{0} \cong$ $A e / N e$ so that we have $L_{0} \subset L_{3} \subset L_{1}+L_{2}$. Since $L_{1} \cap L_{2}=L_{0}$, it follows directly that $L_{3} \cap L_{1}=L_{3} \cap L_{2}=L_{0}$. Clearly $L_{1}+L_{2}=L_{1}+L_{3}=$ $L_{2}+L_{3}$. Hence the ideal lattice of $A$ contains the projective root

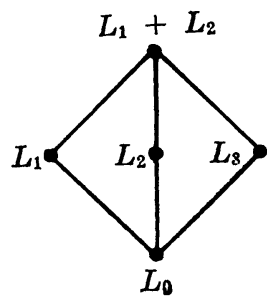

which contradicts the assumption that the lattice is distributive. Thus, $A$ is generalized uni-serial and of finite type.

4. Lattices with vertex of order four. In this section we assume that the center of the endomorphism ring of each simple $A$ module is infinite.

THEOREM. If the ideal lattice of $A$ contains a vertex $V$ of order greater than three such that for some primitive idempotent $e \in A$, the image $V e$ of $V$ is a vertex of order greater than three in the submodule lattice of Ae, then $A$ is of strongly unbounded module type. 
Proof. There exists an ideal $B_{0} \subseteq A$ with distinct covers $B_{1}, B_{2}$, $B_{3}, B_{4}$ such that $B_{i} e \supset B_{0} e, i=1,2,3,4$. Since $A / B_{0}$ modules are $A$ modules we can assume that $B_{0}=0$. Because of the theorem of $\S 1$, we assume that the ideal lattice of $A$ is distributive and hence that

$$
\sum_{i=1}^{4} B_{i}=\bigoplus \sum_{i=1}^{4} B_{i}
$$

There exist primitive idempotents $f_{i} \in A$ such that $f_{i} B_{i} e \neq 0, i=1,2,3,4$. Let $A \subset e A e$ be a set of representatives for the nonzero cosets of the center of $e A e / e N e$. Choose $u_{i}=f_{i} u_{i} e \neq 0 \in B_{i} e, i=1,2,3,4$. For $\lambda \in \Lambda$ we have $A f_{i} / N f_{i} \cong A u_{i} \cong A u_{i} \lambda, i=1,2,3,4$. For each positive integer $n$ define

$$
X^{n}=\bigoplus \sum_{i=1}^{2 n} \varepsilon_{i}(A e)
$$

and denote the socle of $X^{n}$ by $Y^{n}$. Define

$$
\begin{gathered}
T_{\lambda}^{n}=\left\{\sum_{i=1}^{n} \varepsilon_{i}\left(a_{i} u_{1}+c_{i} u_{3}+d_{i} u_{4} \lambda+d_{i-1} u_{4}\right)+\varepsilon_{i+n}\left(b_{i} u_{2}+c_{i} u_{3}+d_{i} u_{4}\right):\right. \\
\left.d_{0}=0, a_{i}, b_{i}, c_{i}, d_{i} \in A, i=1, \cdots, n \cdot\right\}, \\
H_{\lambda}^{n}=X^{n} / T_{\lambda}^{n},
\end{gathered}
$$

and

$$
S_{\lambda}^{n}=Y^{n} / T_{\lambda}^{n}
$$

Since the composition length of $T_{\lambda}^{n}$ is equal to $4 n$ and the composition length of $Y^{n}$ is greater than or equal to $8 n$, the composition length of $S_{\lambda}^{n}$ increases without bound as $n$ increases.

Let $\lambda \neq \mu$ be elements of $\Lambda$. We next prove that $H_{\lambda}^{n}$ and $H_{\mu}^{n}$ are not isomorphic. Suppose $\theta$ is an isomorphism from $H_{\lambda}^{n}$ onto $H_{\mu}^{n}$. Since $X^{n}$ is projective, $\theta$ can be lifted to a endomorphism $\bar{\theta}$ of $X^{n}$. There exist $x_{1}, \cdots, x_{2 n}, y_{1}, \cdots, y_{2 n}$ in $e A e$ such that

$$
\bar{\theta} \varepsilon_{2 n}(e)=\sum_{i=1}^{2 n} \varepsilon_{i}\left(x_{i}\right)
$$

and

$$
\bar{\theta} \varepsilon_{n}(e)=\sum_{i=1}^{2 n} \varepsilon_{i}\left(y_{i}\right)
$$

Since, $\theta \pi_{\lambda} \varepsilon_{n}\left(u_{4}\right) \neq 0$, we have

$$
\pi_{\mu}\left(\sum_{i=1}^{2 n} \varepsilon_{i}\left(u_{4} y_{i}\right)\right)=\theta \pi_{\lambda} \varepsilon_{n}\left(u_{4}\right) \neq 0 .
$$


Thus, since $u_{4} \in M$, there exists $k, 1 \leqq k \leqq 2 n$, such that

$$
y_{k} \notin e N e \text {. }
$$

Since $u_{1} y_{i} \in A u_{2}+A u_{3}+A u_{4}$ for $i>n$, we have $u_{1} y_{i}=0$ for $i>n$, and hence, since $e A e / e N e$ is a division ring, $y_{i} \in e N e$, for $i>n$. Similarly, $\bar{\theta} \varepsilon_{2 n}\left(u_{2}\right) \in T_{\mu}^{n}$ implies $x_{i} \in e N e$, for $i \leqq n$. It follows that

$$
\bar{\theta}\left(\varepsilon_{n} u_{3}+\varepsilon_{2 n} u_{3}\right)=\sum_{i=1}^{n} \varepsilon_{i}\left(u_{3} y_{i}\right)+\sum_{i=n+1}^{2 n} \varepsilon_{i}\left(u_{3} x_{i}\right) \in T_{\mu}^{n} .
$$

Therefore, $u_{3} y_{i}=u_{3} x_{i+n}$ for $i=1, \cdots, n$, and hence,

$$
y_{i}=x_{i+n},(\bmod e N e) \quad i=1, \cdots, n .
$$

From this we obtain

$$
\bar{\theta}\left(\varepsilon_{n}\left(u_{4} \lambda\right)+\varepsilon_{2 n}\left(u_{4}\right)\right)=\sum_{i=1}^{n} \varepsilon_{i}\left(u_{4} \lambda y_{i}\right)+\sum_{i=n+1}^{2 n} \varepsilon_{i}\left(u_{4} y_{i-n}\right) \in T_{\mu}^{n} .
$$

Hence, using the definition of $T_{\mu}^{n}$ there exist $d_{1}, \cdots, d_{n} \in A$ such that

$$
\begin{aligned}
& u_{4} \lambda y_{1}=d_{1} u_{4} \mu, \\
& u_{4} \lambda y_{j}=d_{j} u_{4} \mu+d_{j-1} u_{4}, \quad j=2, \cdots, n,
\end{aligned}
$$

and

$$
u_{4} y_{j}=d_{j} u_{4}, \quad j=1, \cdots, n .
$$

Replacing $d_{j} u_{4}$ by $u_{4} y_{j}$ in these equations, we have

$$
u_{4} \lambda y_{1}=u_{4} y_{1} \mu
$$

and

$$
u_{4} \lambda y_{j}=u_{4} y_{j} \mu+u_{4} y_{j-1}, \quad j=2, \cdots, n .
$$

Since $u_{4} \in M$, a simple induction shows that

$$
y_{i} \in e N e, \quad i=1, \cdots, n .
$$

We conclude that $H_{\lambda}^{n}$ and $H_{\mu}^{n}$ are not isomorphic.

Next, suppose that $H_{\lambda}^{n}$ decomposes and let $\eta$ be an idempotent endomorphism of $H_{\lambda}^{n}$ such that $\eta \pi_{\lambda}\left(\varepsilon_{n}\left(u_{3}\right)\right) \neq 0$. Since $X$ is projective, $\eta$ can be lifted to an endomorphism $\bar{\eta}$ of $X^{n}$. There exist $y_{i j} \in e A e$ such that $\bar{\eta}\left(\varepsilon_{j}(e)\right)=\sum_{i=1}^{2 n} \varepsilon_{i}\left(y_{i j}\right)$. If $j \leqq n$, we have

$$
\bar{\eta}\left(\varepsilon_{j} u_{1}\right)=\sum_{i=1}^{2 n} \varepsilon_{i}\left(u_{1} y_{i j}\right) \in T_{\lambda}^{n}
$$

and hence

$$
y_{i j}=0, \quad(\bmod e N e) \quad 1 \leqq i \leqq n, n+1 \leqq j \leqq 2 n .
$$


For $j \leqq n$, we have,

$$
\bar{\eta}\left(\varepsilon_{j}\left(u_{3}\right)+\varepsilon_{j+n}\left(u_{3}\right)\right)=\sum_{i=1}^{n} \varepsilon_{i}\left(u_{3} y_{i j}\right)+\sum_{i=n+1}^{2 n} \varepsilon_{i}\left(u_{3} y_{i, j+n}\right) \in T_{\lambda}^{n} .
$$

Thus, by the definition of $T_{\lambda}^{n}$,

$$
y_{i j}=y_{i+n, j+n} \quad(\bmod e N e), \quad 1 \leqq i, j \leqq n .
$$

We infer that

$$
\bar{\eta}\left(\varepsilon_{n}\left(u_{4} \lambda\right)+\varepsilon_{2 n}\left(u_{4}\right)\right)=\sum_{i=1}^{n} \varepsilon_{i}\left(u_{4} \lambda y_{i n}\right)+\sum_{i=n+1}^{2 n} \varepsilon_{i}\left(u_{4} y_{i-n, n}\right) \in T_{\lambda}^{n} .
$$

Hence, there exist $d_{0}, \cdots, d_{n} \in A, d_{0}=0$, such that

$$
u_{4} \lambda y_{j n}=d_{j} u_{4} \lambda+d_{j-1} u_{4},
$$

and

$$
u_{4} y_{j n}=d_{j} u_{4}, \quad j=1, \cdots, n .
$$

Replacing $d_{j} u_{4}$ by $u_{4} y_{j n}$, we have

$$
u_{4} \lambda y_{1 n}=u_{4} y_{1 n} \lambda
$$

and

$$
u_{4} \lambda y_{j n}=u_{4} y_{j n} \lambda+u_{4} y_{j-1, n}, \quad j=2, \cdots, n .
$$

Hence, for $i<n$ we obtain $y_{i n}=0(\bmod e N e)$. And, since $\eta$ is idempotent and $e A e / e N e$ is a division ring, $y_{n n}=e(\bmod e N e)$. Now suppose $k<n$. Then

$$
\begin{aligned}
& \bar{\eta}\left(\varepsilon_{k}\left(u_{4} \lambda\right)+\varepsilon_{k+1}\left(u_{4}\right)+\varepsilon_{k+n}\left(u_{4}\right)\right) \\
& =\sum_{i=1}^{n} \varepsilon_{i}\left(u_{4} \lambda y_{i k}+u_{4} y_{i, k+1}\right)+\sum_{i=n+1}^{2 n} \varepsilon_{i}\left(u_{4} y_{i-n, k}\right) \in T_{\lambda}^{n} .
\end{aligned}
$$

Hence, there exist $d_{1}^{k}, d_{2}^{k}, \cdots, d_{n}^{k} \in A, d_{0}^{k}=0$, such that

$$
u_{4} \lambda y_{i k}+u_{4} y_{i, k+1}=d_{j}^{k} u_{4} \lambda+d_{j-1}^{k} u_{4},
$$

and

$$
u_{4} y_{j k}=d_{j}^{k} u_{4}, \quad j=1, \cdots, n .
$$

Replacing $d_{j}^{k} u_{4}$ by $u_{4} y_{j k}$ we obtain $u_{4} y_{1, k+1}=0$, and $u_{4} y_{j, k+1}=u_{4} y_{j-1, k}$, $j=2, \cdots, n, k=1, \cdots, n-1$. It follows from these equations that $y_{1 k}=0,(\bmod e N e)$ for $k=2, \cdots, n$, and $y_{j k}=y_{j+1, k+1}(\bmod e N e), j, k=$ $1, \cdots, n-1$. If $i<j \leqq n$, then

$$
y_{i j}=y_{i-1, j-1}=\cdots=y_{1, j-i+1}=0(\bmod e N e) \text {. }
$$


And, if $n \geqq i \geqq j$,

$$
y_{i j}=y_{i-1, j-1}=\cdots=y_{i-j+1,1}(\bmod e N e) .
$$

These results imply

$$
y_{i j}= \begin{cases}0(\bmod e N e), & \text { if } i<j, \text { or } j \leqq n<i, \\ e(\bmod e N e), & \text { if } i=j, \\ y_{i-j+1,1}(\bmod e N e), & \text { if } j<i \leqq n \text {, or } n<j<i .\end{cases}
$$

We shall now show that the restriction of $\eta$ to $S_{\lambda}^{n}$ is a monomorphism and that $\eta\left(S_{\lambda}^{n}\right)=S_{\lambda \text {. }}^{n}$ Suppose that $x \in Y^{n}$ is such that $\pi_{\lambda}(s)$ is an element of the kernel of $\eta$,

$$
x=\sum_{i=1}^{2 n} \varepsilon_{i}\left(x_{i}\right) .
$$

We have $\eta \pi_{\lambda}(x)=\pi_{\lambda} \bar{\eta}(x)=0$, and so

$$
\begin{aligned}
\bar{\eta}(x) & \in T_{\lambda}^{n} . \\
\bar{\eta}(x) & =\sum_{j=1}^{2 n} \bar{\eta} \varepsilon_{j}\left(x_{j}\right) \\
& =\sum_{j=1}^{2 n} \sum_{i=1}^{2 n} \varepsilon_{i}\left(x_{j} y_{i j}\right) \\
& =\sum_{j=1}^{n} \sum_{i=1}^{n} \varepsilon_{i}\left(x_{j} y_{i-j+1,1}\right)+\sum_{j=n+1}^{2 n} \sum_{i=j}^{2 n} \varepsilon_{i}\left(x_{j} y_{i-j+1,1}\right) .
\end{aligned}
$$

Thus, there exist $a_{1}, b_{i}, c_{i}, d_{i}, i=1, \cdots, n$ in $A, d_{0}=0$ such that

$$
\sum_{j=1}^{i} x_{j} y_{i j}=a_{i} u_{1}+c_{i} u_{3}+d_{i} u_{4} \lambda+d_{i-1} u_{4}
$$

and

$$
\sum_{j=1}^{i} x_{n+j} y_{i j}=b_{i} u_{2}+c_{i} u_{3}+d_{i} u_{4}, \quad \text { for } i=1,2, \cdots, n \text {. }
$$

Using the definition of $T_{\lambda}^{n}$, it follows that

$$
x_{j}=\alpha_{j} u_{1}+\gamma_{j} u_{3}+\delta_{j} u_{4} \lambda+\delta_{j-1} u_{4},
$$

and

$$
x_{j+n}=\beta_{j} u_{2}+\gamma_{j} u_{3}+\delta_{j} u_{4}, \quad j=1, \cdots, n-1,
$$

where $\alpha_{1}=a_{1}, \beta_{1}=b_{1}, \gamma_{1}=c_{1}, \delta_{0}=0, \delta_{1}=d_{1}$, and

$$
\begin{aligned}
& \alpha_{k} u_{1}=a_{k} u_{1}-\alpha_{k-1} u_{1} y_{21}-\cdots-\alpha_{1} u_{1} y_{k 1} \\
& \beta_{k} u_{2}=b_{k} u_{2}-\beta_{k-1} u_{2} y_{21}-\cdots-\beta_{1} u_{2} y_{k 1} \\
& \gamma_{k} u_{3}=c_{k} u_{3}-\gamma_{k-1} u_{3} y_{21}-\cdots-\gamma_{1} u_{3} y_{k 1} \\
& \delta_{k} u_{1}=d_{k} u_{4}-\delta_{k-1} u_{4} y_{21}-\cdots-\delta_{1} u_{4} y_{k 1}, \quad \text { for } k>1 .
\end{aligned}
$$


Hence, $\pi_{\lambda}(x)=0$, and the restriction of $\eta$ to $S_{\lambda}^{n}$ is a monomorphism. The proof can now be completed as in $\S 1$.

\section{BIBLIOGRAPHY}

1. G. Birkhoff, Lattice theory, Amer. Math. Soc. Coll. Publ., Volume XXV, 1948.

2. C. W. Curtis and J. P. Jans, On algebras with a finite number of indecomposable modules, Trans. Amer. Math. Soc. 114 (1965), 122-132.

3. C. W. Curtis and I. Reiner, Representation theory of finite groups and associative algebras, Interscience, New York, 1962.

4. J. P. Jans, On the indecomposable representations of algebras, Ann. of Math. (2) 66 (1957), 418-429.

5. T. Nakayama, Note on uni-serial and generalized uniserial rings, Proc. Imp. Acad. Tokyo 16 (1940), 285-289.

6. H. Tachikawa, Note on algebras of unbounded representation type, Proc. Japan Acad. 36 (1960), 59-61.

Received July 30, 1965. This paper consists of part of the author's doctoral thesis.

The Boeing Company and

THE UNIVERSITY OF WASHINGTON 



\section{PACIFIC JOURNAL OF MATHEMATICS}

\section{EDITORS}

\author{
H. SAMELSON \\ Stanford University \\ Stanford, California \\ J. P. JANS \\ University of Washington \\ Seattle, Washington 98105
}

\author{
J. DUGUNDJI \\ University of Southern California \\ Los Angeles, California 90007 \\ RICHARD ARENS \\ University of California \\ Los Angeles, California 90024
}

\section{ASSOCIATE EDITORS}
E. F. BECKENBACH
B. H. NeumanN
F. WOLF
K. YOSIDA

\section{SUPPORTING INSTITUTIONS}

\author{
UNIVERSITY OF BRITISH COLUMBIA \\ CALIFORNIA INSTITUTE OF TECHNOLOGY \\ UNIVERSITY OF CALIFORNIA \\ MONTANA STATE UNIVERSITY \\ UNIVERSITY OF NEVADA \\ NEW MEXICO STATE UNIVERSITY \\ OREGON STATE UNIVERSITY \\ UNIVERSITY OF OREGON \\ OSAKA UNIVERSITY \\ UNIVERSITY OF SOUTHERN CALIFORNIA
}

STANFORD UNIVERSITY

UNIVERSITY OF TOKYO

UNIVERSITY OF UTAH

WASHINGTON STATE UNIVERSITY

UNIVERSITY OF WASHINGTON

AMERICAN MATHEMATICAL SOCIETY CHEVRON RESEARCH CORPORATION TRW SYSTEMS

NAVAL ORDNANCE TEST STATION 


\section{Pacific Journal of Mathematics}

\section{Vol. 19, No. 1 \\ May, 1966}

A. R. Brodsky, The existence of wave operators for nonlinear equations... 1

Gulbank D. Chakerian, Sets of constant width................... 13

Robert Ray Colby, On indecomposable modules over rings with minimum condition....................................... 23

James Robert Dorroh, Contraction semi-groups in a function space ....... 35

Victor A. Dulock and Harold V. McIntosh, On the degeneracy of the Kepler

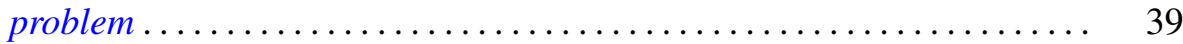

James Arthur Dyer, The inversion of a class of linear operators ......... 57

N. S. Gopalakrishnan and Ramaiyengar Sridharan, Homological dimension of Ore-extensions ................................. 67

Daniel E. Gorenstein, On a theorem of Philip Hall ................. 77

Stanley P. Gudder, Uniqueness and existence properties of bounded observables..................................... 81

Ronald Joseph Miech, An asymptotic property of the Euler function ....... 95

Peter Alexander Rejto, On the essential spectrum of the hydrogen energy and related operators ............................... 109

Duane Sather, Maximum and monotonicity properties of initial boundary

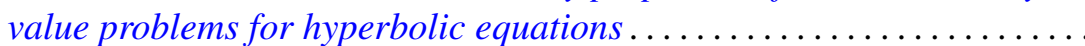

Peggy Strait, Sample function regularity for Gaussian processes with the parameter in a Hilbert space ........................... 159

Donald Reginald Traylor, Metrizability in normal Moore spaces ........... 175

Uppuluri V. Ramamohana Rao, On a stronger version of Wallis' formula ...............................

Adil Mohamed Yaqub, Some classes of ring-logics....... 